\title{
Evaluation of Current Density in DC Motor Brushes for Mining Machines Based on Air Gap Field Measurement
}

\author{
G. Mirzaeva, R.E. Betz and T.J. Summers \\ School of Electrical Engineering and Computer Science \\ University of Newcastle, Australia, 2308
}

\begin{abstract}
Due to robustness and simplicity of control, DC machines continue to be widely used in many applications including mining. Though a mature technology, DC machines still require research attention to accomodate for heavier loads and harder operation conditions dictated by production needs. Recent advances in sensoring, data acquisition and signal processing technologies allow for direct measurement of quantities that used to be theoretically modeled or indirectly measured in the past. Within a research project with industry partners, the research team obtained the measurements of flux densities inside the motor air gap under both static and dynamic conditions. A variety of valuable information about the motor performance can be derived from these measurements. This paper focuses on one particular aspect of the commutation performance of DC machines, namely, the magnitudes and uniformity of current density through a brush, which to a large degree dictate wear rates of the DC motor commutating group. The paper revisits the classical commutation theory, presents a mathematical model for current density estimation across a brush, and applies this model to the experimental results obtained. The results are then discussed and related to the commutation quality of the machine.
\end{abstract}

\section{INTRODUCTION}

Due to development of digital drives and power electronics technologies in the last decades, as well as to the well-known advantages of AC machines, the AC drive technology has become the technology of choice in many industrial applications. However, DC machines continue to be employed in particular demanding applications where the higher power end of electronic devices is combined with extremely dynamical load and harsh operation conditions, such as electrical digging machines (rope shovels and draglines). According to our survey, for a big mining company in Australia it is typical to have around $80 \%$ of DC machines in their motor fleet. Transition to AC technology is happening in these applications as well, however, one can expect that DC machines will continue to be used with the production critical mining equipment for a few more decades.

Though a mature technology, DC machines still require research attention to accomodate for heavier loads and harder operation conditions dictated by production needs. As the bucket size increases and the digging cycle reduces, more challenges are needed to be addressed to ensure efficient and reliable motor operation. Brush and commutation wear continues to be one of the main cause of downtime, both for scheduled maintenance and in reaction to failures.

Under increasingly demanding operation conditions, more fundamental understanding of the issues involved in the commutator/brush wear needs to be gained. One possible approach is to carry out experiments on small DC machines in a laboratory environment. A research project of this type would give valuable results but may not capture the particular wear mechanisms inherent to the large machines in the production environment. To ensure accuracy and applicability of the results to mining machines, it is desirable for the experiments to be carried out on full scale machines under carefully controlled conditions. A big research project has been initiated between the authors of this paper and industry partners that included construction of a facility for dynamic testing of full size industrial motors under controlled conditions.

\section{A. Test facility}

The test facility includes two DC motors connected back-toback, each with a digital control system, converter cabinet and power infrastructure as per a typical rope shovel installation. Both motors are of similar size (around 2000HP) and of types commonly used in the rope shovel application. At any moment in time one motor simulates one of the digging motors (hoist, crowd or swing), the other performs the role of the corresponding dynamic load. Both motors are similarly instrumented by a large number of sensors installed internally (e.g. Hall effect flux sensors and resistive thermal devices) and externally (e.g. differential voltage probes, Hall effect current sensors, Rogowski coil current sensors). The sensors are connected to a Data Acquisition (DAQ) System that includes two separate PCs with high bandwidth ADC cards. A block diagram of the DAQ system appears in Fig. 1. More details on the facility structure and features can be found in [1].

A particular emphasis of this project is placed on direct measurement of flux density inside the motor air gap in both circumferential and longitudial directions. One can see from Fig. 1 that both motors are instrumented by 47 sensors 


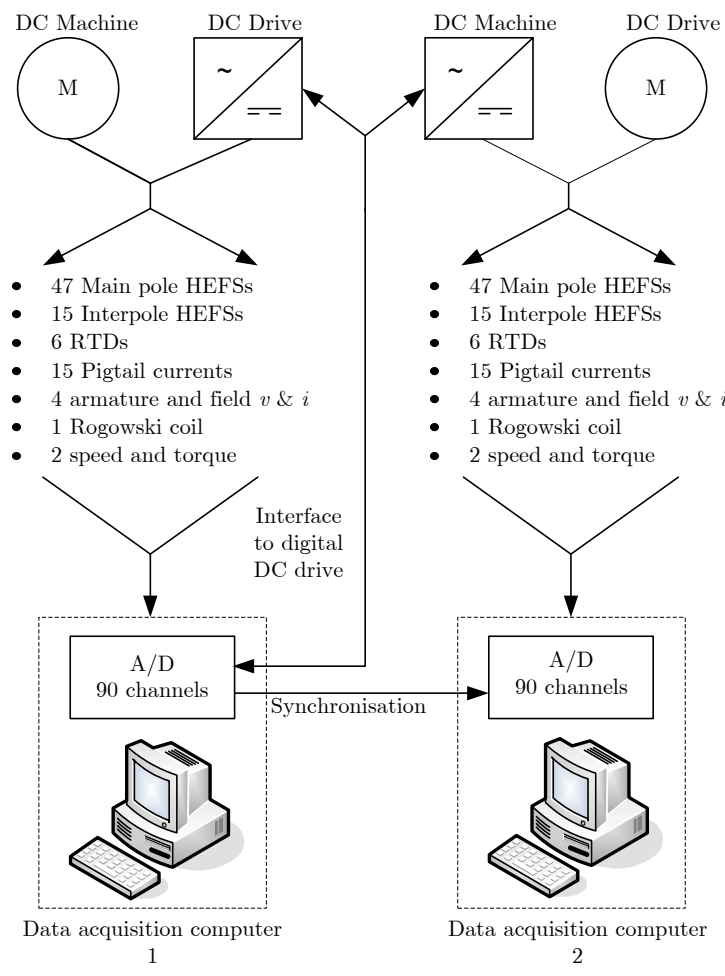

Fig. 1: Block diagram of the data acquisition system

mounted on main poles and 15 sensors mounted on interpoles. These are Melexis MLX90251 Programmable Linear Hall Effect Flux Sensors (HEFS) chosen for their high sensitivity range (measurement of up to 1.8 Tesla), high bandwidth $(23 \mathrm{KHz})$, reasonable accuracy (12 bit), and 2 nd order thermal compensation in the temperature range from $-40^{\circ} \mathrm{C}$ to $150^{\circ} \mathrm{C}$.

The direct measurement of flux distribution in the motor air gap provides intimate details of the motor performance including commutation. The relation between the flux density distribution and the DC motor commutation performance will be explained in the next chapter.

\section{B. Experimental results}

Experimental results obtained for one of the test motors are shown in Fig. 2 and Fig. 3. The measured data is shown in the left column, and the processed data is shown in the right column of both figures. The processing of data included filtering out the $300 \mathrm{~Hz}$ ripple in the armature current, armature voltage and interpole flux, as well as some low pass filtering of the main pole flux signals. The source of the $300 \mathrm{~Hz}$ ripple is the 6-pulse silicon controlled rectifier (SCR). The oscillations seen in the main pole flux signal are due to the armature slot effect: as the armature slot passes the flux sensor, the effective air gap would increase and the flux density would reduce. Given the very small size of the air gap, its variation due to the slot effect was very well pronounced in the experimental results.

In the test illustrated by Fig. 2 the motor was commanded a constant speed of $227 \mathrm{rpm}$, and the load was changed in steps from 0 to 2500A. In the test illustrated by Fig. 3 a step change of load from zero to the rated value of 1925A was applied at each of the speed values that varied from 0 to $625 \mathrm{rpm}$.

From the experimental results obtained, a variety of performance characteristics of the test DC machine can be determined. This paper specifically focuses on one particular aspect of the commutation performance of DC machines, namely, the magnitudes and uniformity of current density through a brush.

The rest of the paper is organised as follows: in the next section the DC machine commutation theory will be revisited and a model for current density profile across a brush will be presented. This model will be then applied to selected fragments of the experimental data of Fig. 2 and Fig. 3 to develop the current density profiles under conditions of interest. These conditions include: steady state with perfect DC signals; steady state in the presence of the $300 \mathrm{~Hz}$ ripple resulting from the SCR drive; and step changes from zero to maximumin load and back to zero. The results will be then discussed and related to the DC motor commutation performance. The paper will be then concluded by the summary of the main ideas and contributions.

\section{Commutation Revisited}

\section{A. Brush wear mechanisms}

Accelerated wear and damage of the brush and the commutator remain one of the biggest issues that limit the DC machine service life. According to [2], two main physical phenomena predominate in the carbon brush wear process: mechanical friction and electrical erosion. Current and current density through the brush play an important role in the both wear mechanisms.

An appropriate brush grade is selected based on average current density that is normally estimated as:

$$
j=\frac{I_{a}}{0.5 N_{b r} T W}
$$

where $j \triangleq$ surface current density; $I_{a} \triangleq$ armature current; $N_{b r} \triangleq$ total number of brushes; $T \triangleq$ thickness of the brush (dimension that runs across the commutator bar and can span 1 or more bars); $W \triangleq$ width of the brush (dimension that runs along the commutator bar).

However, the appropriate average current density ratings of the brush do not guarantee its good performance. It is important that an approximately uniform current distribution is maintained, both between brushes and across each brush. Unevenly shared current results in a commutator film stripping off and a selective brush wear. Once started, selectivity gets worse quickly as resistance of carbon decreases with increase of temperature. After a part of the brush surface area is worn or damaged then the current is distributed in the remaining part with higher current per unit surface area, which further accelerates the brush wear process.

Another important brush wear mechanism is electrical erosion acting via vaporization of carbon with little physical residue. High level of erosion is commonly associated with 

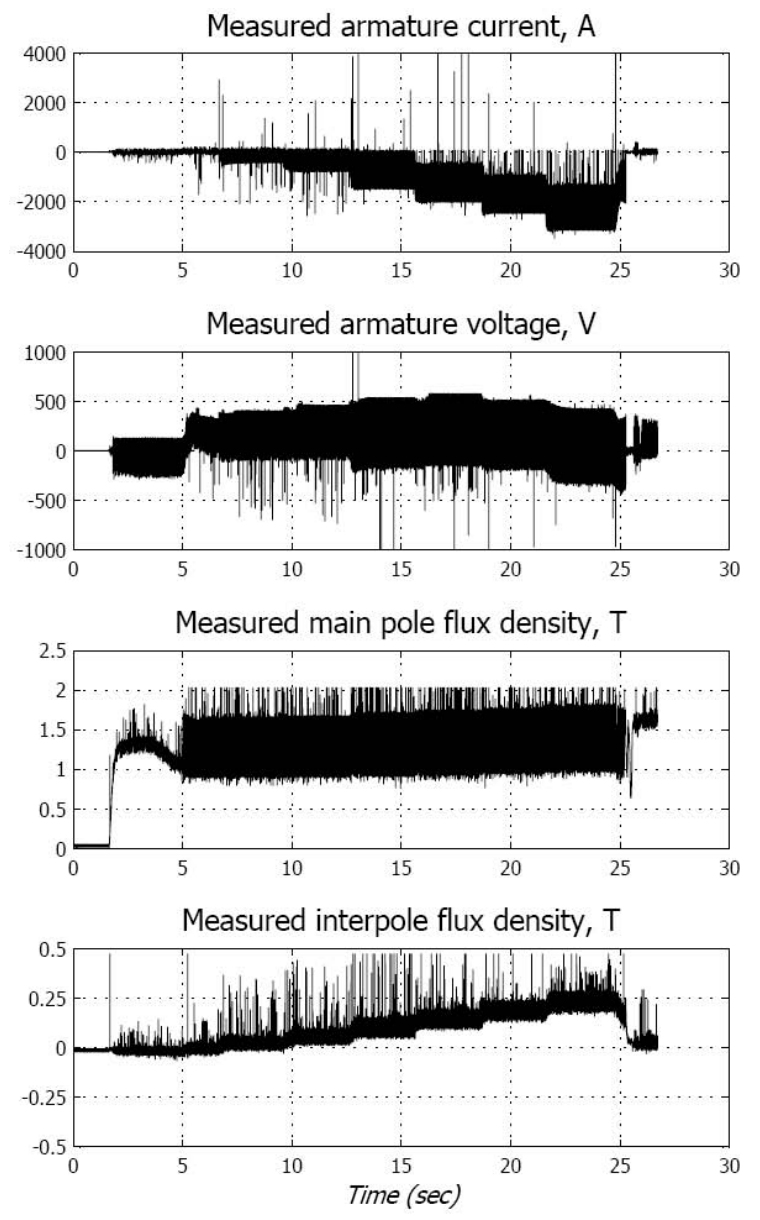
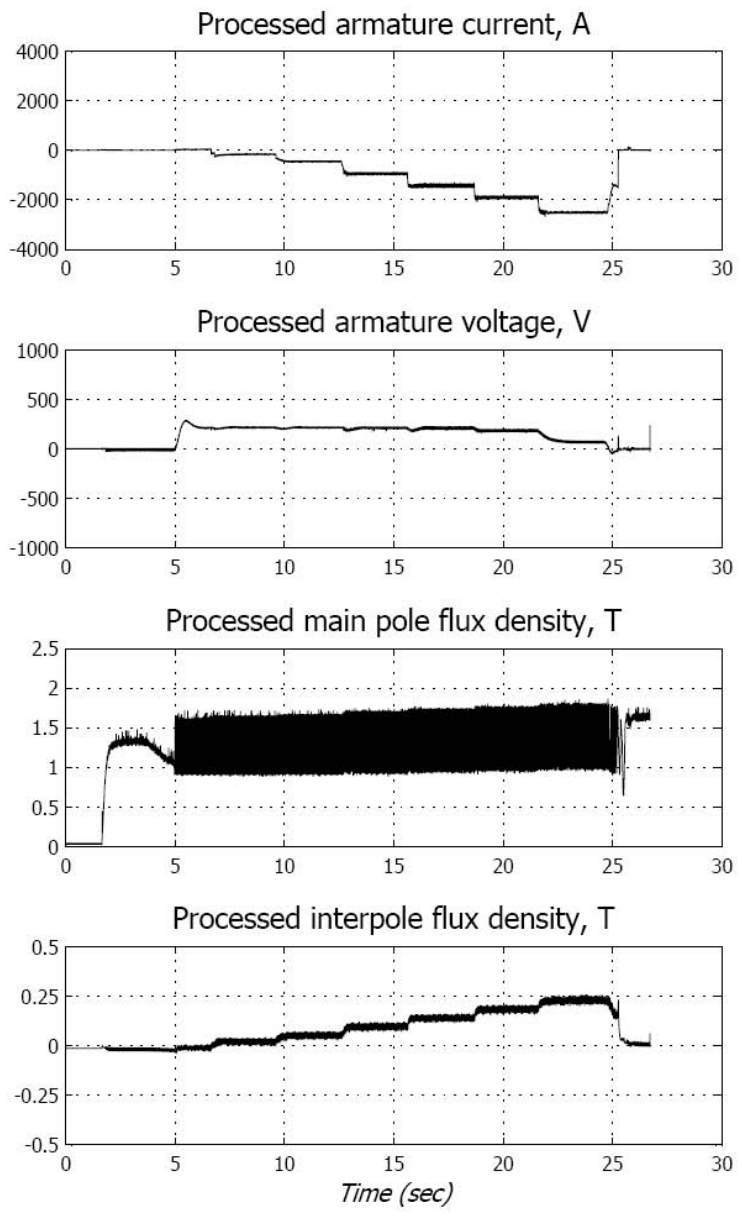

Fig. 2: Application of variable load under constant speed

sparking, which can be due to mechanical as well as electromagnetic issues. Electromagnetic conditions of sparking, according to [3], can be expressed in terms of either critical potential difference or critical released energy, both being related to current distribution across the brush. Therefore, variation of current density is an important measure of inclination of the brush/commutator contact to spark.

\section{B. Classical commutation theory}

In this subsection we will briefly revisit the basics of commutation theory to provide the grounds for the current density model development. For more information a reader is referred to classical text books on DC machines, for example, [4] or [5].

Fig. 4 illustrates current reversal in a coil undergoing commutation. A commutation period $\left(T_{c}\right)$ is the time that it takes from a brush to run off one commutator bar and completely settle on another. During this time the current in the coil undergoing commutation reverses its direction. Fig. 4 shows some intermediate position of the brush (a shaded rectangular) between two commutator bars (rectangulars marked as 1 and 2). Applying Kirchoff's Law to the coil undergoing commutation results in:

$$
U_{A}-U_{B}=i_{1} r_{b r 1}-i_{2} r_{b r 2}=\Sigma e
$$

where $U_{A}-U_{B}$ is the potential difference between points $A$ and $B ; i_{1}, i_{2}$ are currents through parts of the brush that contact commutator bars 1 and 2 respectively; $r_{b r 1}, r_{b r 2}$ are resistances of the corresponding parts of the brush; $\Sigma e \triangleq$ sum of all emfs acting on the coil between points $A$ and $B$.

The abovementioned emfs include:

- A reactive emf $e_{r}$ that is due to the rate of change of current, and is related to the emf of the coil selfinductance $e_{L}$ and the emf due to the mutual inductance $\left(e_{M}\right)$ if the brush spans several commutator bars:

$$
e_{r}=e_{L}+e_{M}=-L_{c} \frac{d i}{d t}-M \frac{d i}{d t}
$$

where $i$ is the current through the coil undergoing commutation; $L_{c}$ is the self inductance of this coil; $M$ is the mutual inductance with the adjacent commutating coils.

- A motional emf $e_{m}$ which is due to the relative motion of the coil conductors with respect to the externally established magnetic field. To a first approximation it can be assumed proportional to the magnetic flux density:

$$
e_{m}=2 l_{c} n_{c} v_{a} B_{c}
$$



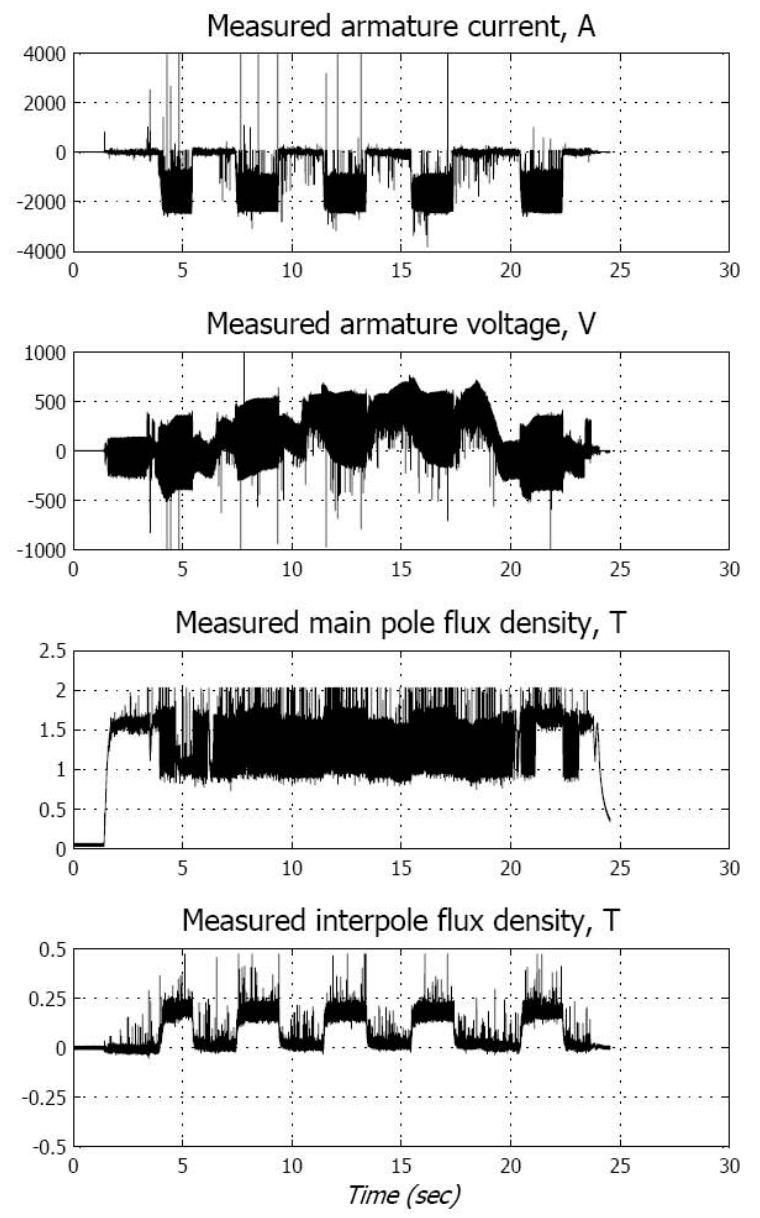
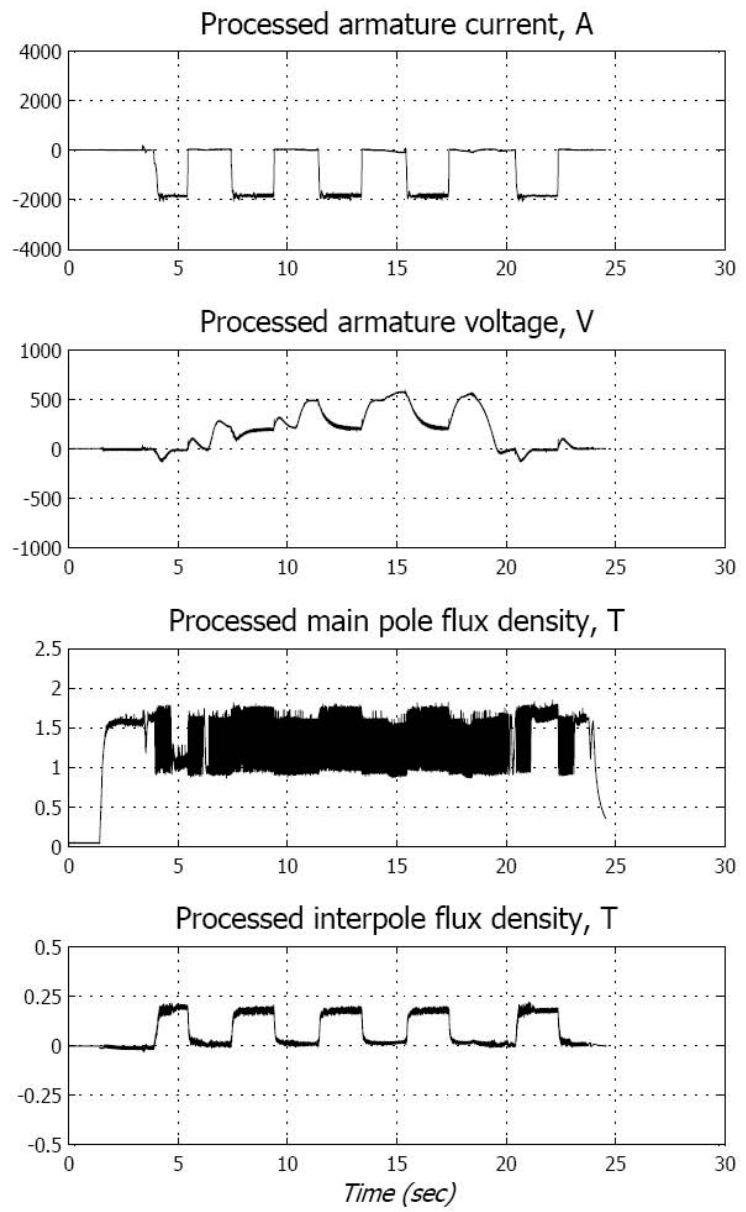

Fig. 3: Application of full load steps at variable speed

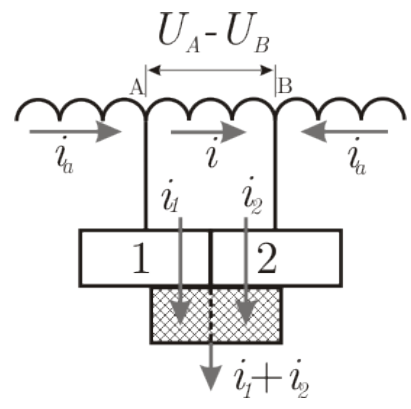

Fig. 4: Currents in the coil undergoing commutation

where $l_{c} \triangleq$ part of the coil length that crosses flux lines of the commutation field; $n_{c} \triangleq$ number of turns in the coil; $v_{a} \triangleq$ linear speed of the armature periphery; $B_{c} \triangleq$ flux density of the total commutation field. $B_{c}=B_{a r}+$ $B_{i p}+B_{c w}$, where $B_{a r}, B_{i p}$ and $B_{c w}$ are flux densities for the armature reaction field, the interpole field and the compensating winding field respectively.

It is clear from Fig. 4 that $i_{1}=i_{a}-i$ and $i_{2}=i_{a}+i$, where $i_{a}$ is the armature current through one current path of the winding. Then it can be derived from (2) that current through the coil undergoing commutation is given by:

$$
\begin{array}{r}
i(\tau)=-i_{a}(1-2 \tau)-i_{c}(\tau) ; \quad i_{c}(\tau)=\frac{\Sigma e}{R_{b r}(\tau)} ; \\
R_{b r}(\tau)=r_{b r}\left(\frac{1}{\tau}+\frac{1}{1-\tau}\right)
\end{array}
$$

where $i_{c}$ is the additional "commutation" current; $\tau$ is time variable normalised to the period of commutation, $\tau=t / T_{c}$; $R_{b r}(\tau)$ is the equivalent resistance of the brush contact to the current $i_{c}$.

If all emfs acting on the coil are compensated $(\Sigma e=0)$ then $i_{c}=0$ and linear commutation takes place. If $\Sigma e<0$ then one has a retarded commutation and if $\Sigma e>0$ then one has an accelerated commutation. Note that, regardless of the type of commutation, the following expressions are true:

$$
\begin{aligned}
& i(\tau)=-i_{a}+\int_{0}^{\tau}\left(\frac{d i}{d \tau}\right) d \tau \\
& i_{1}(\tau)=i_{a}-i(\tau)=2 i_{a}-\int_{0}^{\tau}\left(\frac{d i}{d \tau}\right) d \tau ; \\
& i_{2}(\tau)=i_{a}+i(\tau)=\int_{0}^{\tau}\left(\frac{d i}{d \tau}\right) d \tau
\end{aligned}
$$




\section{Current density model}

Taking the above theoretical basis a few steps further allows one to develop a mathematical model to estimate current density through the brush as a function of normalised time $\tau$ and normalised coordinate $x$ across the brush (i.e. along its thickness dimension).

Firstly, a differential equation can be developed for the current through the armature coil undergoing commutation. Equations (5) are combined and further manipulated in the following way:

$$
\begin{aligned}
i(\tau) & =-i_{a}(1-2 \tau)-\frac{\Sigma e}{R_{b r}(\tau)} \\
& =-i_{a}(1-2 \tau)-\frac{\tau(1-\tau)}{r_{b r}}\left(e_{r}+e_{m}\right)
\end{aligned}
$$

Then substituting $e_{r}=-\frac{L_{c}}{T} \frac{d i}{d \tau}$ for the reactive emf and assuming the motional emf to be a function of the normalised time $e_{m}(\tau)$, and further manipulating, yields:

$$
\frac{d i}{d \tau}+\frac{i_{a}(1-2 \tau)+i}{\tau(1-\tau)} \frac{T r_{b r}}{L_{c}}=\frac{T}{L_{c}} e_{m}(\tau)
$$

This equation can be solved for a given $e_{m}(\tau)$. Then from equations (6) to (8) one can obtain the currents $i(\tau), i_{1}(\tau)$ and $i_{2}(\tau)$. Based on these currents, one can determine current densities through the parts 1 and 2 of the brush (that overlap the commutator bars 1 and 2 respectively) as:

$$
\begin{aligned}
& j_{1}(\tau)=\frac{i_{1}(\tau)}{S_{b r 1}(\tau)}=\frac{i_{1}(\tau)}{S_{b r}(1-\tau)} ; \\
& j_{2}(\tau)=\frac{i_{2}(\tau)}{S_{b r 2}(\tau)}=\frac{i_{2}(\tau)}{S_{b r} \tau}
\end{aligned}
$$

For convenience we then introduce a normalised coordinate $x=d / T_{b r}$ where $d$ is the distance from the leading edge of the brush and $T_{b r}$ is the brush thickness. Realizing that under the assumptions made, the points with $x \leq \tau$ belong to part 2 and the points with $x>\tau$ belong to part 1 of the brush, one can write:

$$
j(x, \tau)= \begin{cases}j_{2}(\tau) & \text { for } 0 \leq x \leq \tau, \\ j_{1}(\tau) & \text { for } \tau<x \leq 1 .\end{cases}
$$

Expression (14) shows how current density in any point across the brush changes in time during the commutation period. Furthermore it is possible to find the average current density over the commutation period in any point across the brush as:

$$
j(x)=\int_{0}^{1} j(x, \tau) d \tau=\int_{0}^{x} j_{1}(\tau) d \tau+\int_{x}^{1} j_{2}(\tau) d \tau
$$

To solve the differential equation for the current through the coil one needs to know the motional emf $e_{m}(\tau)$ which, according to (4) is proportional to the externally established magnetic field in the commutation zone. More accurately, it follows from Faraday's Law that the part of the induced emf related to motion equals to:

$$
e_{m}=2 l_{c} n_{c} v_{a}\left(B_{c}(\theta) \cos \theta+\frac{d B_{c}(\theta)}{d \theta} \sin \theta\right)
$$
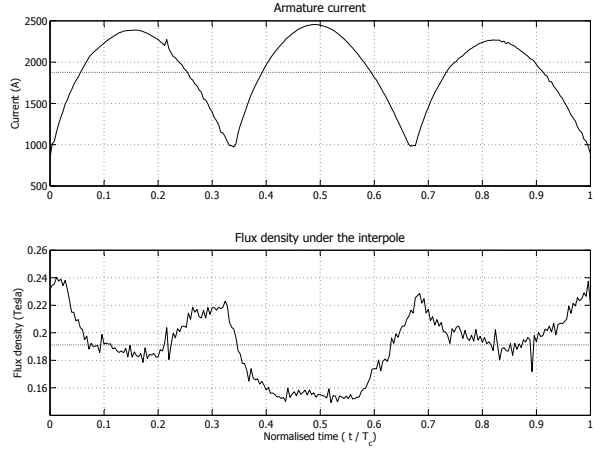

Fig. 5: Armature current and interpole flux density in steady state - fragment of experimental data
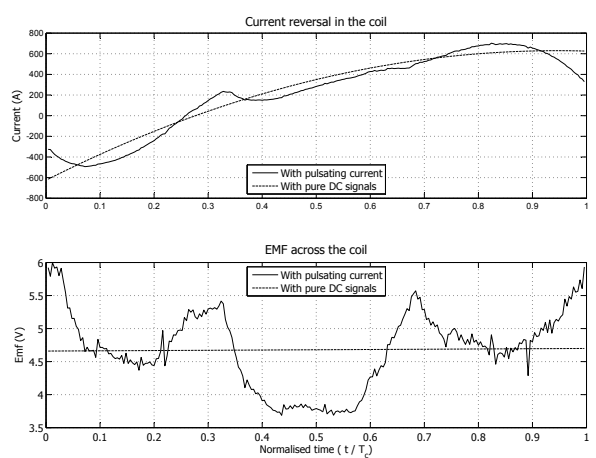

Fig. 6: Current and emf in the coil undergoing commutation for steady state conditions

where $\theta$ is the angular position of the moving conductor relative to the middle of the interpole. Due to small thickness of the interpole, the second term in (16) is usually neglected, and the approximate version (4) is used. In either case, $e_{m}(\tau)$ can be derived from resulting flux density distribution and the position of the coil conductors in the commutation zone. The directly measured flux density in the air gap presents the necessary information for accurate $e_{m}(\tau)$ estimation and application of the explained current density model.

\section{MODEL APPLICATION TO EXPERIMENTAL RESULTS}

The model described by equations (11) to (16) was implemented in the Matlab/ Simulink ${ }^{\circledR}$ environment. The actual parameters, sizes and design details of the armature, the commutator and the brush were included in the implementation. Fragments (one commutation period long) of experimental data from Fig. 2 and Fig. 3 for the armature current and the interpole flux density recorded at the same time were input to the model. Given the relation (16) and other known conditions of the experiment, equations (11) to (13) were solved by the Simulink model. Its output data was post processed by the Matlab procedure to solve the equations (14) and (15) and to plot the results.

Three particular conditions for the DC motor operation were investigated, as presented below. 


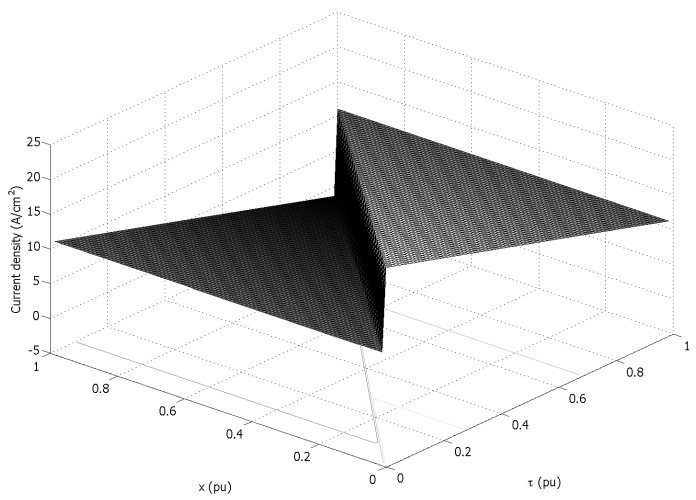

(a) For pure DC current

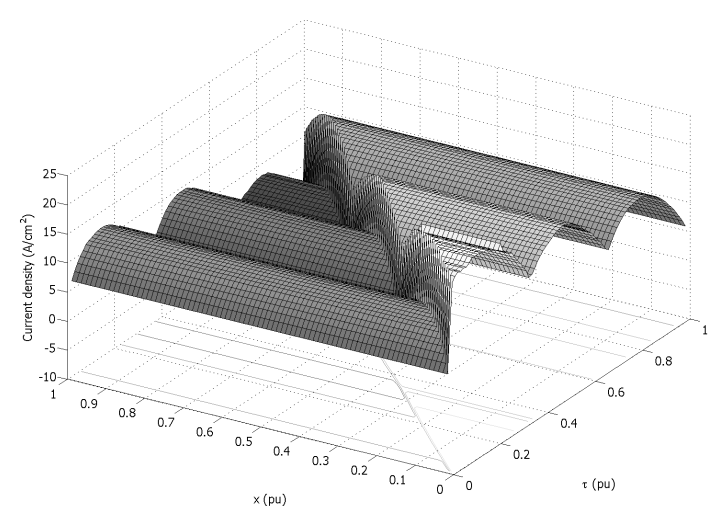

(b) For pulsating current

Fig. 7: Current density profile across the brush and in time for steady state conditions

\section{A. Steady state with pure DC signals}

As a baseline for comparison, a case was modeled when the DC motor is fed by a pure DC source with the current magnitude equal to the average of the pulsating current waveform recorded in the experiment. In practice, this case would correspond to the DC motor fed from a Ward-Leonard system (as implemented with vast majority of the mining draglines). The corresponding time average of the interpole flux density recording was also used with the model. The pure DC (average) armature current and interpole flux are shown in Fig. 5 by dotted lines. Note that the average armature current is at its approximately rated value for the test motor.

The results of the model application are shown in Fig. 6, Fig. 8, Fig. 9 by dotted lines, and in Fig. 7a. From Fig. 6 one can see that the current reversal is not linear. Current changes faster at the beginning than at the end of the commutation, hence the commutation is of the accelerated nature. The emf across the commutating coil is dominated by the motional emf given by (4) and averages to $4.7 \mathrm{~V}$. According to [3] it should not be above $4 \mathrm{~V}$ but this general recommendation does not take into account a special (particularly robust) brush grade used in the mining DC machines.

The current density profile in time and in space, $j(x, \tau)$ from (14), is shown in Fig. 7a. A steep transition between

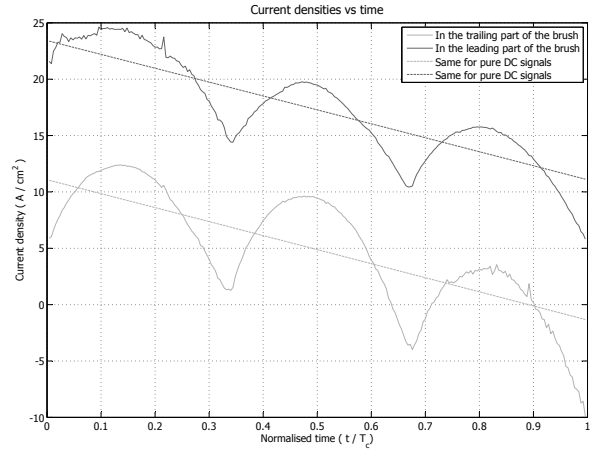

Fig. 8: Current densities in two parts of the brush in time for steady state conditions

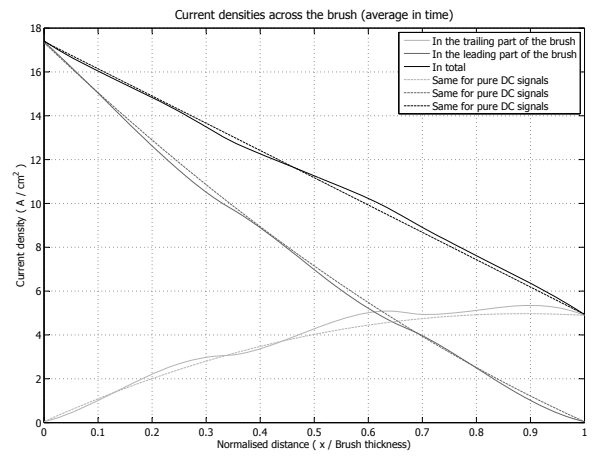

Fig. 9: Average in time current densities across the brush for steady state conditions

the current densities in the two parts of the brush seen in Fig. 7a will be much smoother in real situations, however, as our intension is to average the current densities in time, this should not lead to significant errors.

A 2D plot of the two current densities, $j_{1}(\tau)$ and $j_{2}(\tau)$ from (12) and (13) respectively, allows one to appreciate the defference between a higher current density in the leading part of the brush and a lower current density in its trailing part. The time averages (over a commutation period) and the resulting average current density profile across the brush, $j(x)$ from (15), are shown in Fig. 9. One can see that the average current density is not uniform (as per expression (1)) across the brush and is higher at the leading than at the trailing edge. At the leading edge of the brush the current density is close to (but still under) the maximum value of $20 \mathrm{~A} / \mathrm{cm}^{2}$ for the chosen brush grade.

As a general conclusion, the test motor is slightly overcompensated but still within the permissible margins, and would pass the commonly used steady state tests, such as black band test and brush potential test [3]. Though the DC motor in the rope shovel application operates in all four quadrants, it experiences more load when hoisting than when dropping the bucket, hence it is likely that the brush will be wearing more intensively on one side (the leading side when motoring). 


\section{B. Steady state with pulsating waveforms}

Due to application of the SCR drive, the actual waveforms have a $300 \mathrm{~Hz}$ ripple on top of the DC current values. Examples of the recorded current and interpole flux waveforms are shown in Fig. 5 by solid lines. A form factor (peak to average current) of 1.3 observed in Fig. 5 is a typical value for this type of rectifiers. Similar discussion to that given in the previous subsection applies to Fig. 6, Fig. 8, Fig. 9 (solid lines), and Fig. $7 \mathrm{~b}$. Below are the main observations made in comparison between with the pure DC current case.

The current reversal in the commutating coil shown in Fig. 6 by the solid line follows the dotted line with some deviations. The emf across the commutating coil is distorted too, and in some points in time reaches $6 \mathrm{~V}$. The ripple seen in the armature current waveform is also present in the current densities vs time plots of Fig. 8 and Fig. 7b. The time average current densities across the brush are very close to those for the pure DC current case, as can be seen from Fig. 9.

\section{Step change of the load}

A step change of the load from zero to the rated value and back (see Fig. 3) would normally take more than one commutation period due to the current slew rate limits applied in the drive. The interpole flux would follow the dynamics of the armature current with some delays due to Eddie currents in the interpole pole piece. Experiments have shown that the delay between the interpole flux and the armature current when rising from zero $\left(\Delta t_{\text {rise }}\right)$ is smaller than the corresponding delay when falling from an initially high armature current value $\left(\Delta t_{\text {fall }}\right)$

In this paper we consider two interesting fragments (one commutation period long each), of the experimental data from Fig. 3: (a) when the armature current is close to reaching the rated value while the interpole flux is still building up due to the delay $\Delta t_{\text {rise }}$; and (b) when the armature current is reaching zero but the interpole flux is still at some non-zero value due to the delay $\Delta t_{\text {fall }}$. These fragments, corresponding to a very low rotation speed, are shown in Fig. 10a and Fig. 11a, respectively. The same characteristics as in the previous subsections were obtained for the both cases and are presented in Fig. 10b-c-d and Fig. 11b-c-d.

In the case of the rising edge of the load step (Fig. 10), the fact that the interpole flux rise is delayed plays a positive role for commutation. From comparison between Fig. 10d and Fig. 9, with virtually the same armature current being commutated, the current densities across the brush for the load step (rising edge) are lower than for the steady state condition $\left(14.5 \mathrm{~A} / \mathrm{cm}^{2}\right.$ vs $17.2 \mathrm{~A} / \mathrm{cm}^{2}$ at the leading edge and 6.5 $\mathrm{A} / \mathrm{cm}^{2}$ vs $5.0 \mathrm{~A} / \mathrm{cm}^{2}$ at the trailing edge, respectively). The physical reason for the lower current densities is the fact that the delay in the interpole flux negates, to some degree, its overcompensation.

The opposite is true for the falling edge of the load step illustrated by Fig. 11. The delayed in the interpole flux dynamics with respect to the armature current means that, for a short period of time, the motor is even more overcompensated than in steady state. From Fig. 11a, the current at the beginning of the commutation period is around $2 / 3$ of the current in the steady state example (Fig. 5), and the current at the end of the commutation period is zero. Because of the time averaging, the current densities across the brush (Fig. 11d) are lower than in the steady state example $\left(9.5 \mathrm{~A} / \mathrm{cm}^{2}\right.$ vs $17.2 \mathrm{~A} / \mathrm{cm}^{2}$ at the leading edge and $-0.5 \mathrm{~A} / \mathrm{cm}^{2}$ vs $5.0 \mathrm{~A} / \mathrm{cm}^{2}$ at the trailing edge of the brush, respectively) but the difference between them is higher.

If the drop of the load continued in the negative direction then, for a short period of time, the motor would be overcompensated in a wrong direction. If such conditions were repeatedly applied to the motor, then excessive sparking and accelerated brush wear would be a likely consequence.

\section{CONCLUSIONS AND CONTRIBUtions}

The paper explained about the purposes and the structure of the test facility for dynamic testing of full size motors. It showed some experimental results obtained when running tests on the 2000HP DC motor commonly used in mining applications. The paper then presented the mathematical model for estimation of the current density profile across the brush based on the experimental flux measurements. The model was then applied to the experimental results obtained. The results of the model application were explained and related to the motor commutation performance.

The contributions of the paper include:

- Unique recordings of the direct flux density measurements inside the motor air gap;

- Mathematical model for estimation of the current density profile across the brush based on the experimentally measured flux densities;

- Application of this model to explain and predict the commutation performance of the test DC motor.

Possible areas where the presented model can be usefully applied include:

- Interpretation of brush wear patterns;

- Adjustment of the brush axis position;

- Prediction of the brush wear due to electrical effects;

- Adjustments to the interpole field strengths to improve the quality of commutation;

- Allow recommendations to be made for acceptable rates of change of the armature current.

\section{REFERENCES}

[1] G. Mirzaeva, R. Betz, T. Summers, and I. Marxsen, "Development of a unique dc motor test facility in the hunter valley," in Proc. of the Australian Mining Technology Conference: Smart Technologies for Sustaining the Minerals Boom. 16-18 September 2008, Twin Waters, QLD, Australia, 2008, pp. 95-106.

[2] R. Hamilton, "Dc motor brush life," IEEE Transactions on Industry Applications, vol. 36, no. 6, pp. 1682-1687, 2000.

[3] M. Kostenko and L. Piotrovsky, Electrical Machines. Part1. Peace Publishers, 1961, translated from Russian by A.E.Tchernukhin, El.Eng.

[4] A. E. Clayton, The performance and design of direct current machines, 3rd ed. London: Sir Isaac Pitman and Sons Ltd, 1969.

[5] A. E. Clayton, C. K. Jr., and S. D. Umans, Electric machinery, 4th ed. New York: McGraw-Hill, 1983. 

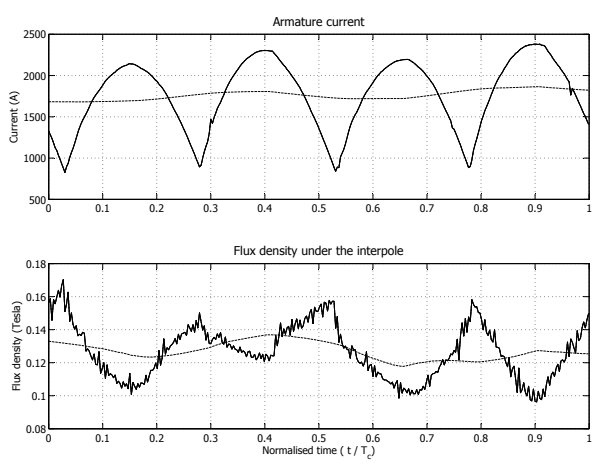

(a) Armature current and interpole flux density
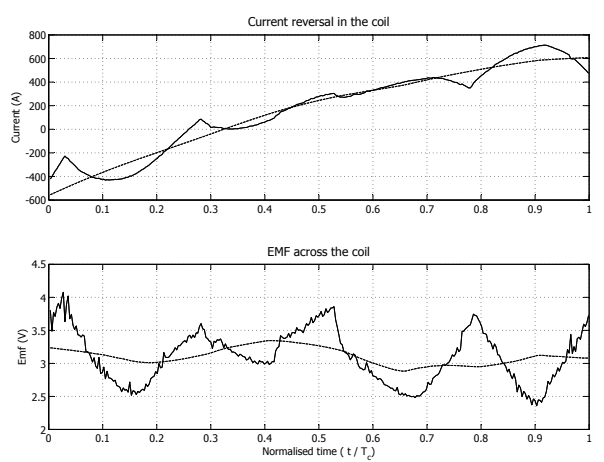

(b) Current and emf in the commutating coil

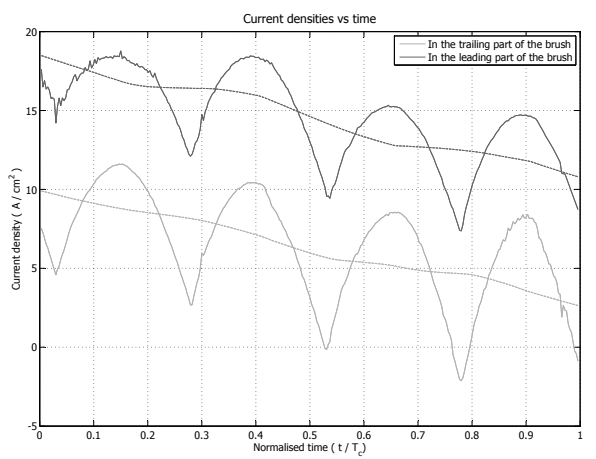

(c) Current densities in time

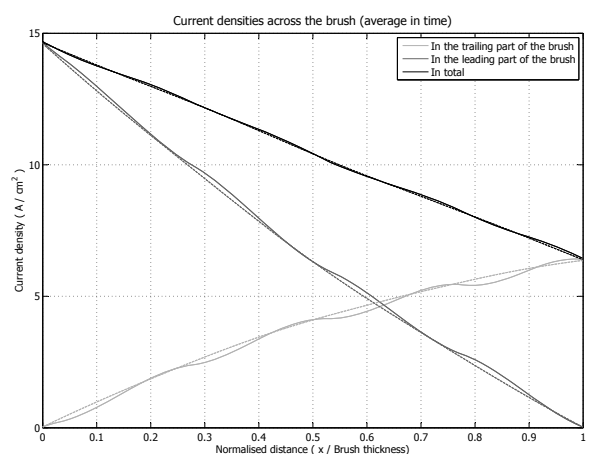

(d) Time average current densities across the brush

Fig. 10: Model application to the load step (falling edge)
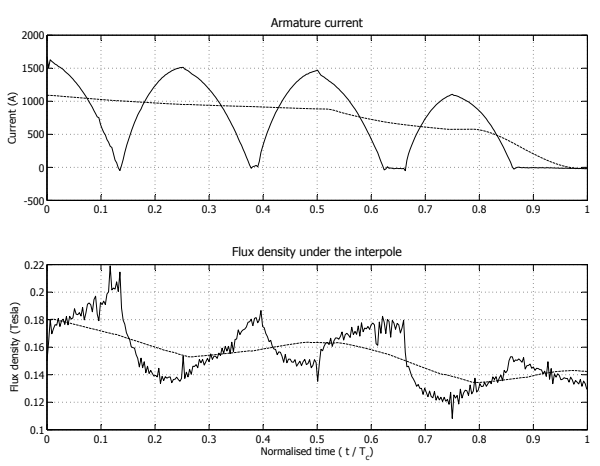

(a) Armature current and interpole flux density
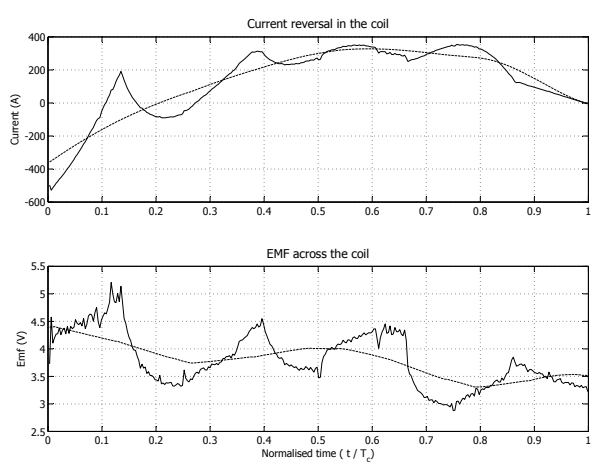

(b) Current and emf in the commutating coil

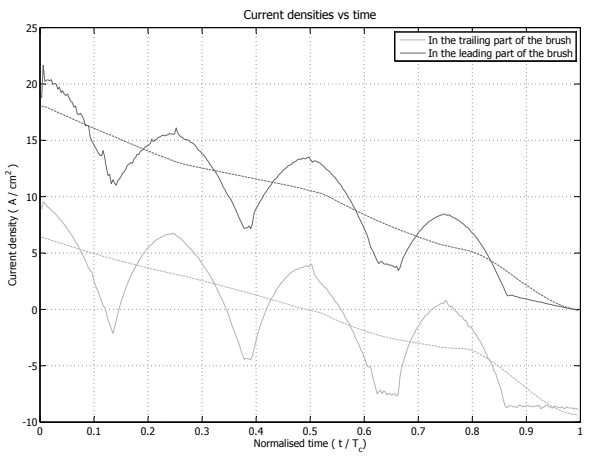

(c) Current densities in time

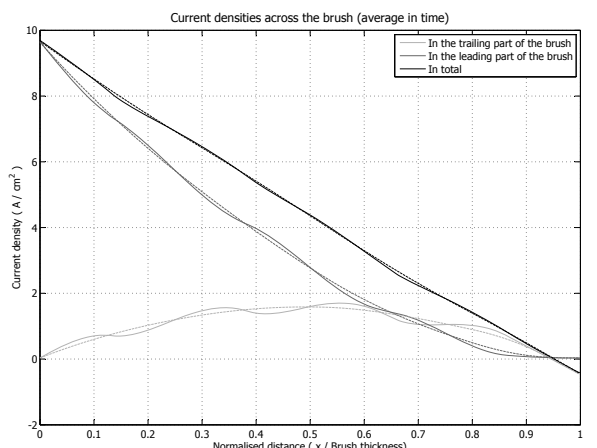

(d) Time average current densities across the brush

Fig. 11: Model application to the load step (falling edge) 